\title{
Green function for a non-Markovian Fokker-Planck equation: Comb-model and anomalous diffusion
}

\author{
L. R. da Silva ${ }^{1}$, A. A. Tateishi ${ }^{2}$, M. K. Lenzi ${ }^{3}$, E. K. Lenzi ${ }^{2}$, and P. C. da Silva ${ }^{1,4}$ \\ ${ }^{1}$ Departamento de Física and National Institute of Science and Technology for Complex Systems, \\ Universidade Federal do Rio Grande do Norte, 59072-970, Natal, RN, Brazil \\ ${ }^{2}$ Departamento de Física and National Institute of Science and Technology for Complex Systems, \\ Universidade Estadual de Maringá, Avenida Colombo, 5790, 87020-900, Maringá, PR, Brazil \\ ${ }^{3}$ Departamento de Engenharia Química, Universidade Federal do Paraná, \\ Setor de Tecnologia - Jardim das Américas, Caixa Postal 19011, 81531-990, Curitiba, PR, Brazil \\ 4 Centro Federal de Educação Tecnológica do Rio Grande do Norte, \\ Av. Sen. Salgado Filho, 1559, 59015-000, Tirol, Natal, RN
}

(Received on 10 February, 2009)

\begin{abstract}
We investigate solutions, by using the Green function approach, for a system governed by a non-Markovian Fokker-Planck equation and subjected to a Comb structure. This structure consists of the axis of structure as the backbone and fingers which are attached perpendicular to the axis. For this system, we consider an arbitrary initial condition, in the presence of time dependent diffusion coefficients and spatial fractional derivative, and analyze the connection to the anomalous diffusion.
\end{abstract}

Keywords: Anomalous diffusion, Comb-model, Fokker-Planck

\section{INTRODUCTION}

The existence of several diffusive processes in which the mean-square displacement is not asymptotically linear in time such as micelles dissolved in salted water [1], surface growth and transport of fluid in porous media [2], two dimensional rotating flow [3], subrecoil laser cooling [4], diffusion on fractals [5], enhanced diffusion in active intracellular transport [6], particle diffusion in a quasi-two-dimensional bacterial bath [7] has motivated the study of several approaches, in particular, the fractional diffusion equations [8-14] due to the broadness of applications. They have been used to investigate, for example, anomalous transport in disordered systems [15], diffusion on fractals [16], and in non-Markovian dynamical processes in protein folding [17]. This variety of applications of the fractional diffusion equations have stimulated the study of several formal aspects such as the behavior at the origin [18], the connection with generalized master equation [19], their solutions[20-31] by taking external force and spatial dependent diffusion coefficient into account, the effect produced by reaction terms [32-34], solutions in confined regions with spatial and time dependent boundary conditions [35, 36], and the relation between the fractional diffusion equations and comb-model [37-42]. In this point, it is also interesting to mention that the comb-model is applied to investigate porous medium related to exploration of low dimensional percolation clusters, the problem of flow transfer in disordered systems [43], electrophoresis process and tumor development [44]. From the above discussion, we note the relevance of these investigations concerning the fractional diffusion equations and the importance of extending these cases to open the wide range of scenarios. In this direction, we dedicated this work to investigate the solutions for an extension of the comb-model which is based on the following non-Markovian diffusion equation:

$$
\begin{aligned}
\frac{\partial}{\partial t} \rho(x, y ; t) & =\int_{0}^{t} d t^{\prime} \mathcal{D}_{y}\left(t-t^{\prime}\right) \frac{\partial^{2}}{\partial y^{2}} \rho\left(x, y ; t^{\prime}\right) \\
& +\delta(y) \int_{0}^{t} d t^{\prime} \mathcal{D}_{x}\left(t-t^{\prime}\right) \frac{\partial^{\mu}}{\partial|x|^{\mu}} \rho\left(x, y ; t^{\prime}\right)
\end{aligned}
$$

where $\mathcal{D}_{y}(t)$ and $\mathcal{D}_{x}(t)$ are time dependent diffusion coefficients and the fractional derivative applied to the spatial variable is the Riesz-Weyl operator [45].

The plan of this work is to investigate Eq. (1) by taking the boundary conditions $\rho( \pm \infty, y, t)=0$ and $\rho(x, \pm \infty, t)=0$ and the initial condition $\rho(x, y, 0)=\widehat{\rho}(x, y)$ into account, where $\widehat{\rho}(x, y)$ is how the system is initially distributed and it is normalized, i.e., $\int_{-\infty}^{\infty} d x \int_{-\infty}^{\infty} d y \widehat{\rho}(x, y)=1$. We first consider the case given by $\mathcal{D}_{y}(t)=\mathcal{D}_{y} \delta(t), \mathcal{D}_{x}(t)=\mathcal{D}_{x} \delta(t)$ and $\mu=2$. The result obtained for this case permits us to show the effect produced by an arbitrary initial condition on the solution. In particular, the spreading of the distribution for initial times where the influence of the initial condition is important. Following, we incorporate a power law dependence for the diffusion coefficients, i.e., $\mathcal{D}_{y}(t)=\mathcal{D}_{y} t^{\gamma_{y}-2} / \Gamma\left(\gamma_{y}-1\right)\left(\mathcal{D}_{y}(s)=\mathcal{D}_{y} s^{1-\gamma_{y}}\right.$ and $\left.0<\gamma_{y}<1\right), \mathcal{D}_{x}(t)=\mathcal{D}_{x} t^{\gamma_{x}-2} / \Gamma\left(\gamma_{x}-1\right)\left(\mathcal{D}_{x}(s)=\mathcal{D}_{x} s^{1-\gamma_{x}}\right.$ and $\left.0<\gamma_{x}<1\right)$. This time dependence for the diffusion coefficients may be related to the fractional time derivatives and it makes possible to obtain the inverse Laplace transform. Another time dependence for the diffusion coefficients is possible, however may lead us to cumbersome calculations. Afterwards, we incorporated spatial fractional derivatives, i.e., $\mu \neq 2$, in our analysis. For these cases, we obtain the exact solution by using the Green function approach [46] and the dispersion relation when it is defined. These developments are presented in Sec. II and in Sec. III we present our discussion and conclusion about the results found here.

\section{NON-MARKOVIAN FOKKER PLANCK EQUATION}

Let us start by considering Eq.(1) with $\mathcal{D}_{y}(t)=\mathcal{D}_{y} \delta(t)$, $\mathcal{D}_{x}(t)=\mathcal{D}_{x} \delta(t)$ and $\mu=2$. For this case, it is given by

$$
\frac{\partial}{\partial t} \rho(x, y, t)=\mathcal{D}_{y} \frac{\partial^{2}}{\partial y^{2}} \rho(x, y, t)+\mathcal{D}_{x} \delta(y) \frac{\partial^{2}}{\partial x^{2}} \rho(x, y, t) .
$$

The singular term in Eq.(2) indicates the diffusion of the system on $x$-direction only occurs in the line $y=0$ which implies that the diffusion coefficient $\mathcal{D}_{x}$ differs from zero only 
in $y=0$. A direct consequence of this restriction on the system is the presence of an anomalous diffusion (subdiffusion) in this direction. This feature may be verified by evaluating the mean square displacement of the solution on the variable $x$.

We analyze Eq.(2) by accomplishing the boundary conditions $\rho( \pm \infty, y, t)=0$ and $\rho(x, \pm \infty, t)=0$ and the initial condition $\rho(x, y, 0)=\widehat{\rho}(x, y)$ with $\int_{-\infty}^{\infty} d x \int_{-\infty}^{\infty} d y \rho(x, y, 0)=$ $\int_{-\infty}^{\infty} d x \int_{-\infty}^{\infty} d y \widehat{\rho}(x, y)=1$, i.e., the distribution is initially normalized. Note that solutions for this equation are obtained by taking a general initial condition into account, in contrast to the cases worked out, for example, in [39-42] which consider particular forms for the initial condition. In order to perform this analysis and obtain the solution for this case, we use integrals transforms (Laplace and Fourier) and use the Green function approach. Applying the Fourier transform on the $x$ variable $\left(\mathcal{F}\{\ldots\}=\int_{-\infty}^{\infty} d x e^{-i k_{x} x} \ldots\right.$ and $\mathcal{F}^{-1}\{\ldots\}=$ $\left.\frac{1}{2 \pi} \int_{-\infty}^{\infty} d k_{x} e^{i k_{x} x} \ldots\right)$ and the Laplace transform on $t$ variable $\left(\mathcal{L}\{\ldots\}=\int_{0}^{\infty} d t e^{-s t} \ldots\right.$ and $\left.\mathcal{L}^{-1}\{\ldots\}=\frac{1}{2 \pi i} \int_{-i \infty+c}^{i \infty+c} d s e^{s t} \ldots\right)$ in Eq.(2), we obtain

$$
\begin{aligned}
\mathcal{D}_{y} \frac{d^{2}}{d y^{2}} \rho\left(k_{x}, y, s\right) & -\left(s+\mathcal{D}_{x} k_{x}^{2} \delta(y)\right) \rho\left(k_{x}, y, s\right) \\
& =-\rho\left(k_{x}, y, 0\right) .
\end{aligned}
$$

This equation may be solved by using the Green function ap- proach [46] which leads us to obtain

$$
\rho\left(k_{x}, y, s\right)=-\int_{-\infty}^{\infty} d \bar{y} \widehat{\rho}\left(k_{x}, y\right) \mathcal{G}\left(k_{x}, y, \bar{y}, s\right)
$$

with the Green function governed by equation

$$
\begin{aligned}
\mathcal{D}_{y} \frac{d^{2}}{d y^{2}} \mathcal{G}\left(k_{x}, y, \bar{y}, s\right) & -\left(s+\mathcal{D}_{x} k_{x}^{2} \delta(y)\right) \mathcal{G}\left(k_{x}, y, \bar{y}, s\right) \\
& =\delta(y-\bar{y})
\end{aligned}
$$

and subjected to the boundary condition $\mathcal{G}\left(k_{x}, \pm \infty, \bar{y}, s\right)=0$. After some calculation, it is possible to show that the solution of Eq.(5) is given by

$$
\begin{aligned}
\mathcal{G}\left(k_{x}, y, \bar{y}, s\right) & =-\frac{1}{2 \sqrt{s \mathcal{D}_{y}}} \\
& \times\left(e^{-\sqrt{\frac{s}{\mathcal{D}_{y}}}|y-\bar{y}|}-e^{-\sqrt{\frac{s}{\mathcal{D}_{y}}}(|y|+|\bar{y}|)}\right) \\
& -\frac{e^{-\sqrt{\frac{s}{\mathcal{D}_{y}}}(|y|+|\bar{y}|)}}{2 \sqrt{s \mathcal{D}_{y}}-\mathcal{D}_{x} k_{x}^{2}} .
\end{aligned}
$$

By performing the inverse of Laplace and Fourier transforms in Eq.(6), we have that

$$
\begin{aligned}
\mathcal{G}(x, y, \bar{y}, t) & =-\frac{\delta(x)}{\sqrt{4 \pi \mathcal{D}_{y} t}}\left(e^{-\frac{(y-\bar{y})^{2}}{4 \mathcal{D}_{y} t}}-e^{-\frac{(|y|+|\bar{y}|)^{2}}{4 \mathcal{D}_{y} t}}\right) \\
& -\frac{1}{\sqrt{8 \mathcal{D}_{x} \sqrt{\mathcal{D}_{y}}}}\left(\frac{|y|+|\bar{y}|}{\sqrt{4 \pi \mathcal{D}_{y}}}\right) \int_{0}^{t} d \bar{t} \frac{e^{-\frac{(|y|+|\bar{y}|)^{2}}{4 \mathcal{D}_{y}(t-\bar{t})}}}{\left[(t-\bar{t}) \bar{t}^{\frac{1}{2}}\right]^{\frac{3}{2}}} \mathrm{H}_{1,1}^{1,0}\left[\sqrt{\frac{2 \sqrt{\mathcal{D}_{y}}}{\mathcal{D}_{x} \sqrt{\bar{t}}}}|x| \begin{array}{c}
\left(\frac{1}{4}, \frac{1}{4}\right) \\
(0,1)
\end{array}\right]
\end{aligned}
$$

where $\mathrm{H}_{p, q}^{m, n}\left[x \mid \begin{array}{l}\left(a_{1}, A_{1}\right), \cdots,\left(a_{p}, A_{p}\right) \\ \left(b_{1}, B_{1}\right), \cdots,\left(b_{q}, B_{q}\right)\end{array}\right]$ is the Fox $\mathrm{H}$ function [47]. The presence of this function in Eq. (7) indicates that the system subjected to the comb structure exhibits anomalous diffusion. This anomalous behavior of the system in the $x$ direction may be evidenced by analyzing the dispersion rela- tion $\sigma_{x}^{2}=\left\langle x^{2}\right\rangle-\langle x\rangle^{2}$ which manifests a subdiffusive behavior (see Eq. (9)). By applying inverse Fourier transform in Eq.(4) and using Eq.(7) it is possible to find the distribution $\rho(x, y ; t)$ and show that

$$
\begin{aligned}
\rho(x, y ; t) & =\int_{-\infty}^{\infty} d \bar{y} \frac{\widehat{\rho}(x, \bar{y})}{\sqrt{4 \pi \mathcal{D}_{y} t}}\left(e^{-\frac{(y-\bar{y})^{2}}{4 \mathcal{D}_{y} t}}-e^{-\frac{(|y|+|\bar{y}|)^{2}}{4 \mathcal{D}_{y} t}}\right) \\
& -\int_{-\infty}^{\infty} d \bar{x} \int_{-\infty}^{\infty} d \bar{y} \int_{0}^{t} d \bar{t} \frac{\widehat{\rho}(\bar{x}, \bar{y})}{\sqrt{8 \mathcal{D}_{x} \sqrt{\mathcal{D}_{y}}}}\left(\frac{|y|+|\bar{y}|}{\sqrt{4 \pi \mathcal{D}_{y}}}\right) \frac{e^{-\frac{(|y|+|\bar{y}|)^{2}}{4 \mathcal{D}_{y} \bar{t}}}}{\left[(t-\bar{t}) \bar{t}^{\frac{1}{2}}\right]^{\frac{3}{2}}} \mathrm{H}_{1,1}^{1,0}\left[\sqrt{\frac{2}{\mathcal{D}_{x}} \sqrt{\frac{\mathcal{D}_{y}}{\bar{t}}}|x-\bar{x}|} \mid \begin{array}{c}
\left(\frac{1}{4}, \frac{1}{4}\right) \\
(0,1)
\end{array}\right] .
\end{aligned}
$$

Note that the first term in Eq.(8) is essentially due to the arbitrary form of the initial condition and depending on the choice of the initial condition it vanishes recovering the results presented in [42]. This additional term for $\rho(x, y ; t)$ 


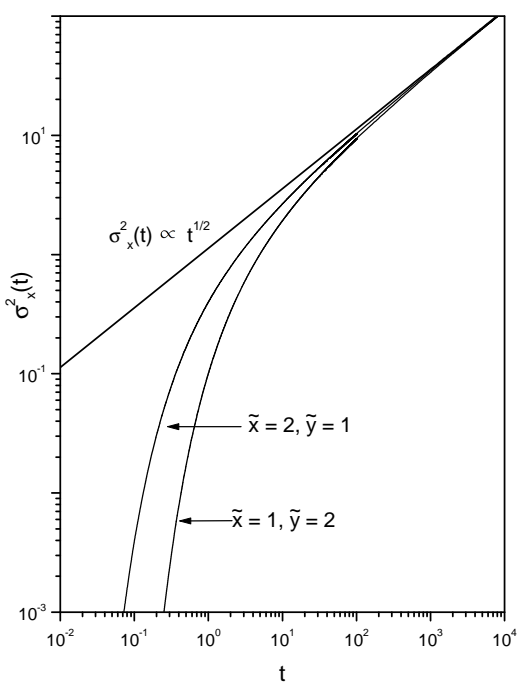

FIG. 1: Behavior of $\sigma_{x}^{2}$ versus $t$ for the initial condition $\widehat{\rho}(x, y)=$ $\delta(x-\tilde{x}) \delta(y-\tilde{y})$ is illustrated for typical values of $\widetilde{x}$ and $\widetilde{y}$. Note that the spreading of the solution may exhibit an initial transient depending on the choice of initial condition, i.e., $\tilde{x}$ and $\tilde{y}$, due to the structure of the system.

may also play an important role to investigate situations characterized by the system not initially localized at the point $(x, y)=(0,0)$, for example, in drug deliver, flow transfer in disordered systems, tumor development or contaminant diffusion in a heterogeneous media. By using the above equation it is possible to find the dispersion relation for $x$ and $y$ directions which are useful to understand the diffusive process produced by the comb-model. By taking the initial condition $\rho(x, y ; 0)=\delta(x-\tilde{x}) \delta(y-\tilde{y})$ into account, we can to show that the dispersion relation for $x$-direction is

$$
\sigma_{x}^{2}=2 \mathcal{D}_{x} \sqrt{\frac{t}{\pi \mathcal{D}_{y}}} e^{-\frac{\tilde{y}^{2}}{4 \mathcal{D}_{y} t}}+|\widetilde{y}| \frac{\mathcal{D}_{x}}{\mathcal{D}_{y}} \operatorname{erfc}\left(\frac{|\widetilde{y}|}{2 \sqrt{\mathcal{D}_{y} t}}\right)
$$

and the dispersion relation for the $y$ variable is $\sigma_{y}^{2}=2 \mathcal{D}_{y} t$ indicating that we have usual diffusive behavior in this direction.

Now, we analyze Eq.(1) with $\mathcal{D}_{y}(t)=\mathcal{D}_{y} t^{\gamma_{y}-2} / \Gamma\left(\gamma_{y}-1\right)$ $\left(\mathcal{D}_{y}(s)=\mathcal{D}_{y} s^{1-\gamma_{y}}\right.$ and $\left.0<\gamma_{y}<1\right), \mathcal{D}_{x}(t)=\mathcal{D}_{x} t^{\gamma_{x}-2} / \Gamma\left(\gamma_{x}-1\right)$ $\left(\mathcal{D}_{x}(s)=\mathcal{D}_{x} s^{1-\gamma_{x}}\right.$ and $\left.0<\gamma_{x}<1\right)$, and $\mu=2$. Notice that this choice for the time dependence of the diffusion coefficients is related to the fractional time derivatives employed to analyze the subdiffusive processes. Other choices for the time dependence of diffusion coefficients are possible, however may lead us to a cumbersome calculations to obtain the inverse Laplace transform. For this case Eq. (1) can be written as

$$
\begin{aligned}
\frac{\partial}{\partial t} \rho(x, y ; t) & =\int_{0}^{t} d t^{\prime} \mathcal{D}_{y}\left(t-t^{\prime}\right) \frac{\partial^{2}}{\partial y^{2}} \rho\left(x, y ; t^{\prime}\right) \\
& +\delta(y) \int_{0}^{t} d t^{\prime} \mathcal{D}_{x}\left(t-t^{\prime}\right) \frac{\partial^{2}}{\partial x^{2}} \rho\left(x, y ; t^{\prime}\right)
\end{aligned}
$$

with $\mathcal{D}_{y}(t)$ and $\mathcal{D}_{x}(t)$ defined above. By taking the previous boundary and initial conditions into account one can show that the solution in the Laplace space is given by

$$
\rho(x, y, s)=-\int_{-\infty}^{\infty} d \bar{x} \int_{-\infty}^{\infty} d \bar{y} \widehat{\rho}(\bar{x}, \bar{y}) \widetilde{\mathcal{G}}(x, \bar{x}, y, \bar{y}, s)
$$

with the Green function satisfying the equation

$$
\begin{aligned}
& \mathcal{D}_{y}(s) \frac{\partial^{2}}{\partial y^{2}} \widetilde{\mathcal{G}}(x, \bar{x}, y, \bar{y}, s)+\delta(y) \mathcal{D}_{x}(s) \frac{\partial^{2}}{\partial x^{2}} \widetilde{\mathcal{G}}(x, \bar{x}, y, \bar{y}, s) \\
& -s \widetilde{\mathcal{G}}(x, \bar{x}, y, \bar{y}, s)=\delta(y-\bar{y}) \delta(x-\bar{x})
\end{aligned}
$$

and subjected to the conditions $\mathcal{G}(x, \bar{y}, \pm \infty, s)=0$ and $\mathcal{G}( \pm \infty, \bar{y}, y, s)=0$. After some calculations, the solution for above equation can be written as follows

$$
\begin{aligned}
& \widetilde{\mathcal{G}}(x, \bar{x}, y, \bar{y} ; s)=-\frac{\delta(x-\bar{x})}{2 \sqrt{s \mathcal{D}_{y}(s)}} \\
& \times\left(e^{-\sqrt{\frac{s}{\mathcal{D}_{y}(s)}}|y-\bar{y}|}-e^{-\sqrt{\frac{s}{\mathcal{D}_{y}(s)}}(|y|-|\bar{y}|)}\right) \\
& -\frac{e^{-\sqrt{\frac{2 \sqrt{s \mathcal{D}_{y}(s)}}{\mathcal{D}_{x}(s)}}|x-\bar{x}|}}{\sqrt{8 \mathcal{D}_{x}(s) \sqrt{s \mathcal{D}_{y}(s)}}} e^{-\sqrt{\frac{s}{\mathcal{D}_{y}(s)}}(|y|+|\bar{y}|)}
\end{aligned}
$$

By using Eq.(11) and Eq.(13), one can find the dispersion relations $\sigma_{x}$ and $\sigma_{y}$ in the Laplace space by considering the initial condition $\widehat{\rho}(x, y)=\delta(x-\widetilde{x}) \delta(y-\widetilde{y})$. For $\sigma_{x}^{2}(s)$ we have that

$$
\sigma_{x}^{2}(s)=\frac{\mathcal{D}_{x}(s)}{\sqrt{s^{3} \mathcal{D}_{y}(s)}} e^{-\sqrt{\frac{s}{\mathcal{D}_{y}(s)}}|\tilde{y}|}
$$

and for $\sigma_{y}^{2}(s)$ we obtain that $\sigma_{y}^{2}(s)=2 \mathcal{D}_{y}(s) / s^{2}$. These results obtained for $\sigma_{x}^{2}(s)$ and $\sigma_{y}^{2}(s)$ show that, depending on the choice of $\mathcal{D}_{x}(s)$ and $\mathcal{D}_{y}(s)$, the spreading of the distribution may exhibit different diffusive behaviors. By performing the inverse Laplace transform in $\sigma_{x}^{2}(s)$ and $\sigma_{y}^{2}(s)$ and taking the time dependence required above for the diffusion coefficients into account we obtain that

$$
\begin{aligned}
\sigma_{x}^{2}(t) & =\frac{\mathcal{D}_{x} t^{\gamma_{x}}}{\sqrt{\mathcal{D}_{y} t_{y}}} \mathrm{H}_{1,1}^{1,0}\left[\frac{|\bar{y}|}{\sqrt{\mathcal{D}_{y} t \gamma_{y}}} \mid \begin{array}{l}
\left(1+\gamma_{x}-\frac{\gamma_{y}}{2}, \frac{\gamma_{y}}{2}\right) \\
(0,1)
\end{array}\right] \\
\sigma_{y}^{2}(t) & =\frac{2 \mathcal{D}_{y} t^{\gamma_{y}}}{\Gamma\left(1+\gamma_{y}\right)} .
\end{aligned}
$$

Note that the dispersion relations obtained for this case indicate that the solution has an anomalous dispersion in both directions and depend on the parameters $\gamma_{x}$ and $\gamma_{y}$. It is also interesting to mention that the dispersion relation obtained for the $y$-direction is the same as the ones obtained for the fractional diffusion equations and the asymptotic behavior of $\sigma_{x}^{2}(t)$ is $t^{\gamma_{x}-\gamma_{y} / 2}$ for long time. In addition, the inverse Laplace of the Green function is given by 


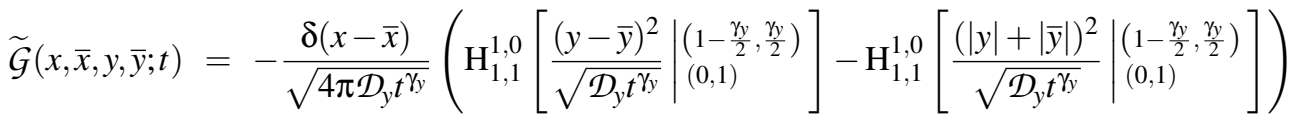

$$
\begin{aligned}
& -\int_{0}^{t} \frac{d \bar{t}}{(t-\bar{t}) \sqrt{8 \mathcal{D}_{x} \bar{t}^{\gamma_{x}} \sqrt{\mathcal{D}_{y} \bar{t}^{\gamma_{y}}}}} \mathrm{H}_{1,1}^{1,0}\left[\left.\sqrt{\frac{2}{\mathcal{D}_{x} \bar{t}^{\gamma_{x}}} \sqrt{\mathcal{D}_{y} \bar{t}^{\gamma_{y}}}}|x-\bar{x}|\right|_{\left(\begin{array}{c}
(\beta, \xi) \\
(0,1)
\end{array}\right]} \mathrm{H}_{1,1}^{1,0}\left[\frac{|y|+|\bar{y}|}{\sqrt{\mathcal{D}_{y} \bar{t}^{\gamma_{y}}}} \mid \begin{array}{c}
\left(0, \frac{\gamma_{y}}{2}\right) \\
(0,1)
\end{array}\right]\right.
\end{aligned}
$$

with $\beta=1-\gamma_{x} / 2-\gamma_{y} / 4$ and $\xi=\gamma_{x} / 2-\gamma_{y} / 4$.

Let us consider Eq.(1) with $\mu \neq 2$. Applying the previous procedure one can show that the solution is given by $\rho(x, y ; t)=\int_{-\infty}^{\infty} d \bar{x} \int_{-\infty}^{\infty} d \bar{y} \widehat{\rho}(\bar{x}, \bar{y}) \overline{\mathcal{G}}(x-\bar{x}, y, \bar{y} ; t)$ with the Green function given by

$$
\begin{aligned}
& \overline{\mathcal{G}}(x, y, \bar{y}, t)=-\frac{\delta(x)}{\sqrt{4 \pi \mathcal{D}_{y} t \gamma_{y}}}\left(\mathrm{H}_{1,1}^{1,0}\left[\frac{(y-\bar{y})^{2}}{\sqrt{\mathcal{D}_{y} t \gamma_{y}}} \mid \begin{array}{l}
\left(1-\frac{\gamma_{y}}{2}, \frac{\gamma_{y}}{2}\right) \\
(0,1)
\end{array}\right]-\mathrm{H}_{1,1}^{1,0}\left[\frac{(|y|+|\bar{y}|)^{2}}{\sqrt{\mathcal{D}_{y} t^{\gamma_{y}}}} \mid \begin{array}{l}
\left(1-\frac{\gamma_{y}}{2}, \frac{\gamma_{y}}{2}\right) \\
(0,1)
\end{array}\right]\right) \\
& -\frac{1}{\pi|x|} \int_{0}^{t} \frac{d \bar{t}}{(t-\bar{t}) \bar{t}^{\frac{\gamma_{y}}{2}}} \mathrm{H}_{2,3}^{2,1}\left[\frac{2 \sqrt{\mathcal{D}_{y} \bar{t}^{\gamma_{y}}}}{\mathcal{D}_{x} \bar{t}^{\gamma_{x}}}|x|^{\mu} \mid \begin{array}{l}
(1,1),(\bar{\beta}, \bar{\xi}) \\
\left(\frac{1}{2}, \frac{\mu}{2}\right),(1,1),\left(1, \frac{\mu}{2}\right)
\end{array}\right] \mathrm{H}_{1,1}^{1,0}\left[\frac{|y|+|\bar{y}|}{\sqrt{\mathcal{D}_{y}(t-\bar{t})^{\gamma_{y}}}} \mid \begin{array}{l}
\left(0, \frac{\gamma_{y}}{2}\right) \\
(0,1)
\end{array}\right]
\end{aligned}
$$

with $\bar{\beta}=1-\gamma_{y} / 2$ and $\bar{\xi}=\gamma_{x}-\gamma_{y} / 2$.

\section{DISCUSSION AND CONCLUSION}

We worked out a non-Markovian Fokker-Planck by taking different scenarios into account, focusing on the comb-like structure. The solutions of this Fokker-Planck equation were obtained by considering the presence of time dependent diffusion coefficients, fractional spatial derivatives, and a general initial condition, which may be regarded as an interesting contribution in this context of comb-like structure. We start our analysis with the case characterized by the diffusion coefficients $\mathcal{D}_{y}(t)=\mathcal{D}_{y} \delta(t)$ and $\mathcal{D}_{x}(t)=\mathcal{D}_{x} \delta(t)$ with $\mu=2$. This case is essentially the cases analyzed, for example, in Refs. $[41,42]$ for a particular initial condition. Here we consider a general initial condition for this case and show that if the system is not initially localized on the line $y=0$ the mean square displacement, variance, of the distribution in $x$-direction has an initially transient before recovering the subdiffusive behavior characterized by $t^{1 / 2}$. In addition, the solution has an addition term which is directly related to the choice of the initial condition. Following, we incorporate a time dependence on the diffusion which may be related to time fractional derivatives. For this case, we also work out in the Laplace space - as general as possible - the solution and the variance in $x$ and $y$ directions. The results show that, depending on the choice of the diffusion coefficients, we may have different diffusive behaviors which remind us of the fractional diffusion equations of distributed order [48-50]. Afterwards, we consider $\mu \neq 2$, i.e., we incorporate a spatial fractional time derivative on $x$-direction. The presence of this spatial fractional derivative on the $x$ indicates that the solution in this direction is characterized by power law distribution which may be related to the Lévy distributions. For this case, as for the cases analyzed before, we obtain the exact solution. Finally, we expect that the results obtained here may be useful to investigate systems where a comb- like structure is present.

\section{ACKNOWLEDGMENTS}

We thank CNPq/INCT-SC and Fundação Araucária for partial financial support.
[1] A. Ott, J. P. Bouchaud, D. Langevin, and W. Urbach, Phys. Rev. Lett. 65, 2201 (1990).

[2] H. Spohn, J. Phys. I France 3, 69 (1993).

[3] T. H. Solomon, E. R. Weeks, and H. L. Swinney, Phys. Rev. Lett. 71, 3975 (1993).

[4] F. Bardou, J. P. Bouchaud, O. Emile, A. Aspect, and C. CohenTannoudji, Phys. Rev. Lett. 72, 203 (1994).

[5] J. Stephenson, Physica A 222, 234 (1995).
[6] A. Caspi, R. Granek, and M. Elbaum, Phys. Rev. Lett. 85, 5655 (2000).

[7] Xiao-Lun Wu and A. Libchaber, Phys. Rev. Lett. 84, 3017 (2000).

[8] R. Hilfer, Applications of Fractional Calculus in Physics (World Scientific, Singapore, 2000).

[9] R. Metzler and J. Klafter, Phys. Rep. 339, 1 (2000).

[10] R. Metzler and T. F. Nonnenmacher, Chem. Phys. 284, 67, 
(2002).

[11] B. J. West, M. Bologna, and P. Grigolini, Physics of Fractal Operators (Springer, New York, 2002).

[12] G. M. Zaslavsky, Phys. Rep. 371, 461 (2002).

[13] J. Klafter, M. F. Shlesinger, and G. Zumofen, Phys. Today 49, 33 (1996)

[14] R. Hilfer, R. Metzler, A. Blumen and and J. Klafter, Strange Kinetics, Chemical Physics, 284 Numbers 1-2 (PergamonElsevier, Amsterdam, 2004).

[15] R. Metzler, E. Barkai, and J. Klafter, Physica A 266, 343 (1999).

[16] D. Campos, V. Méndez, and J. Fort, Phys. Rev. E 69, 031115 (2004).

[17] S. S. Plotkin and P. G. Wolynes, Phys. Rev. Lett. 80, 5015 (1998).

[18] Y. E. Ryabov, Phys. Rev. E 68, 030102 (2003).

[19] R. Metzler, E. Barkai, and J. Klafter, Europhys. Lett, 46, 431 (1999).

[20] W. R. Schneider and W. Wyss, J. Math. Phys. 30, 134 (1989).

[21] F. Mainardi and G. Pagnini, Appl. Math. Comput. 141, 51 (2003).

[22] B. N. N. Achar and J. W. Hanneken, J. Mol. Liq. 114, 147 (2004).

[23] R. Gorenflo, A. Iskenderov and Y. Luchko, Fractional Calculus and Applied Analysis 3, 75 (2000).

[24] Fu-Yao Ren, Jin-Rong Liang, wei-Yuan Qiu, Xiao-Tian Wang, Y. Xu, and R. R. Nigmatullin, Phys. Lett. A 312, 187 (2003).

[25] OM P. Agrawal, Nonlinear Dynamics 29, 145 (2002).

[26] A. Hanyga, Proc. R. Soc. London A 458, 429 (2002).

[27] S. A. El-Wakil and M. A. Zahran, Chaos Solitons \& Fractals 12, 1929 (2001)

[28] S. A. El-Wakil, A. Elhanbaly, and M. A. Zahran, Chaos Solitons \& Fractals 12, 1035 (2001).

[29] E. K. Lenzi, R. S. Mendes, K. S. Fa, L. C. Malacarne, and L. R. da Silva, J. Math. Phys. 44, 2179 (2003).

[30] E. K. Lenzi, R. S. Mendes, J. S. Andrade, L. R. da Silva, and
L. S. Lucena, Phys. Rev. E 71, 052109 (2005).

[31] P. C. Assis da Silva, R. P. de Souza, P. C. da Silva, L. R. da Silva, L. S. Lucena, and E. K. Lenzi , Phys. Rev. E 73, 032101 (2006).

[32] K. Seki, M. Wojcik, and M. Tachiya, J. Chem. Phys. 119, 2165 (2003).

[33] S. B. Yuste, L. Acedo, and K. Lindenberg, Phys. Rev. E 69, 036126 (2004).

[34] I. M. Sokolov, M. G. W. Schmidt, and F. Sagués, Phys. Rev. E 73, 031102 (2006).

[35] R. Rossato, M. K. Lenzi, L. R. Evangelista and E. K. Lenzi, Phys. Rev. E 76, 032102 (2007).

[36] L. S. Lucena, L. R. da Silva, L. R. Evangelista, M. K. Lenzi, R. Rossato, and E. K. Lenzi, Chem. Phys. 344, 90 (2008)

[37] V. E. Arkhincheev, JETP Letters 86, 508 (2007).

[38] I. A. Lubashevski and A. A. Zemlyanov, JETP 87, 700 (1998).

[39] V. E. Arkhincheev, JETP 88, 710 (1999).

[40] A. M. Reynolds, Physica A 334, 39 (2004).

[41] V. E. Arkhincheev, Physica A 280, 304 (2000).

[42] V. E. Arkhincheev, Chaos 17, 043102 (2007).

[43] K. V. Chukbar, JETP 82, 719 (1996).

[44] A. Iomin, J. Phys.: Conf. Ser. 7, 57 (2005).

[45] I. Podlubny, Fractional Differential Equations (Academic Press, San Diego, 1999).

[46] M. P. Morse and H. Feshbach, Methods of Theoretical Physics (McGrawHill, New York, 1953).

[47] A. M. Mathai and R. K. Saxena, The H-function with Application in Statistics and other Disciplines (Wiley Eastern, New Delhi, 1978).

[48] A. V. Chechkin, R. Gorenflo, and I. M. Sokolov, Phys. Rev. E 66, 046129 (2002).

[49] I. M. Sokolov, A. V. Chechkin, and J. Klafter, Acta Phys. Pol. B 35, 1323 (2004)

[50] F. Mainardi and G. Pagnini, J. Comput. Appl. Math. 207, 245 (2007). 